\title{
Glueball mass spectrum from supergravity
}

\author{
Csaba Csáki; Hirosi Ooguri, Yaron $\mathrm{Oz}^{\dagger}$ and John Terning \\ Department of Physics, University of California at Berkeley, \\ Berkeley, CA 94720, and \\ Theoretical Physics Group, Lawrence Berkeley National Laboratory, \\ Mail Stop 50A-5101, Berkeley, CA 94720

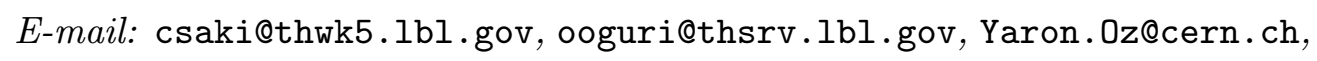 \\ iterning Qaivin.
}

ABSTRACT: We calculate the spectrum of glueball masses in non-supersymmetric Yang-Mills theory in three and four dimensions, based on a conjectured duality between supergravity and large $N$ gauge theories. The glueball masses are obtained by solving supergravity wave equations in a black hole geometry. We find that the mass ratios are in good numerical agreement with the available lattice data. We also compute the leading $\left(g_{Y M}^{2} N\right)^{-1}$ corrections to the glueball masses, by taking into account stringy corrections to the supergravity action and to the black hole metric. We find that the corrections to the masses are negative and of order $\left(g_{Y M}^{2} N\right)^{-3 / 2}$. Thus for a fixed ultraviolet cutoff the masses decrease as we decrease the 't Hooft coupling, in accordance with our expectation about the continuum limit of the gauge theories.

KEYwords: p̈p-brānes, D-brānèsi.

${ }^{*}$ Research fellow, Miller Institute for Basic Research in Science.

${ }^{\dagger}$ Also at: Institute for Theoretical Physics, University of California, Santa Barbara, CA 93106. 


\section{Contents}

ii. Introduction

2. Glueballs in three dimensions

3. Glueballs in four dimensions

望. Leading string theory corrections

5.5. Summary and discussion

6. Note added

\section{Introduction}

Recently Maldacena formulated a conjecture [i] stating that the large $N$ limit of the maximally supersymmetric conformal theories in 3,4 and 6 dimensions are dual to superstring/ $M$ theory on $\mathrm{AdS}_{4} \times \mathbf{S}^{7}, \mathrm{AdS}_{5} \times \mathbf{S}^{5}$ and $\mathrm{AdS}_{7} \times \mathbf{S}^{4}$ respectively, where $\mathrm{AdS}_{d}$ is a $d$-dimensional anti-de Sitter space. More recently Witten proposed [2] that one can extend this duality to non-supersymmetric theories such as pure QCD. In this case the AdS space is replaced by the Schwarzschild geometry describing a black hole in the AdS space. When the curvature of the spacetime is small compared to the string scale and the Planck scale, superstring/ $M$ theory is well-approximated by supergravity. It was found that the supergravity description gives results that are in qualitative agreement with expectations for QCD at strong coupling. This includes the area law behavior of Wilson loops, the relation between confinement and monopole condensation, the existence of a mass gap for glueball states, the behavior of Wilson loops for higher representations, and the construction of heavy quark baryonic states [20,

In this paper, we use the supergravity description of large $N$ gauge theories to compute the scalar glueball mass spectrum explicitly for pure $\mathrm{QCD}_{3}$ and $\mathrm{QCD}_{4}$. The glueball masses in QCD can be obtained by computing correlation functions of gauge invariant local operators or the Wilson loops, and looking for particle poles.

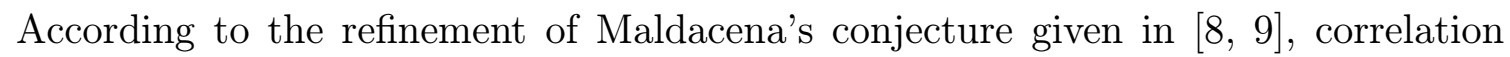
functions of a certain class of local operators (chiral primary operators and their superconformal descendants) are related at large $N$ and large $g_{Y M}^{2} N$ to tree level 
amplitudes of supergravity. The correspondence between the chiral operators and

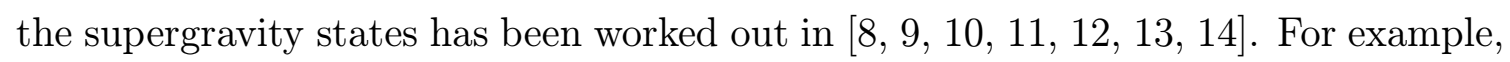
the operator $\operatorname{tr} F^{2}$ in four dimensions corresponds to the dilaton field of supergravity in ten dimensions. Therefore the scalar glueball ${ }^{1} J^{P C}=0^{++}$in QCD which couples to $\operatorname{tr} F^{2}$ is related to the dilaton propagating in the black hole geometry. In particular, its

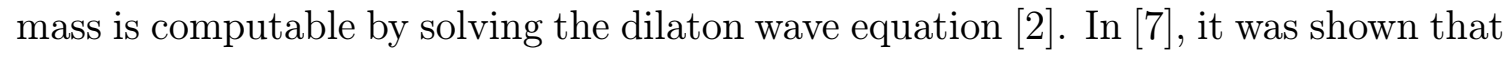
the correlation function of Wilson loops is also expressed in terms of supergraviton exchange if the distance between the loops becomes larger than their sizes, leading again to the supergravity wave equation.

In this paper we will solve the wave equations numerically to obtain the glueball masses. Since this description preserves all the symmetries of QCD, we can identify the spin and the other quantum numbers of the glueballs. The mass ratios turn out to be in excellent agreement with the available lattice data in the continuum limit. This is surprising since a priori the supergravity computations are to be compared with the strong ultraviolet coupling limit of the gauge theory $g_{Y M}^{2} N \gg 1$.

As we will see, the supergravity computation at $g_{Y M}^{2} N \gg 1$ gives the glueball masses in units of the fixed ultraviolet cutoff $\Lambda_{U V}$. For finite 't Hooft coupling $\lambda=g_{Y M}^{2} N$, the glueball mass $M$ would be a function of the form,

$$
M^{2}=f(\lambda) \Lambda_{U V}^{2}
$$

To take the continuum limit $\Lambda_{U V} \rightarrow \infty$, we have to simultaneously take $\lambda \rightarrow 0$ so that the right-hand side of this equation becomes of the order of the QCD mass scale $\Lambda_{Q C D}$. This in particular requires that $f(\lambda)$ decreases as we decrease the 't Hooft coupling $\lambda$.

We compute the leading $\lambda^{-1}$ corrections to the supergravity computation and show that this is indeed the case. On the superstring side, the $\lambda^{-1}$ corrections are due to the finite string tension. The leading order string correction to the low-energy supergravity action was computed in $\left[15_{1}^{5}, 1\right] \overline{1}$. This modifies both the background black hole metric and the supergravity wave equation in that background. Recently the stringy correction to the black hole metric was obtained in [i] modified supergravity equation. We use both this metric and the string corrected wave equation to compute the leading $\lambda^{-1}$ corrections to the $0^{++}$glueball masses in $\mathrm{QCD}_{3}$. We find:

1. The corrections to the masses are negative and of order $\lambda^{-3 / 2}$ :

$$
f(\lambda)=c_{0}+c_{1} \lambda^{-3 / 2}+\cdots, \quad c_{1}<0,
$$

for the ground state and the first 5 excited levels of the $0^{++}$glueball. Thus, for a fixed ultraviolet cutoff, the masses decrease as we decrease the 't Hooft coupling, in accordance with the expectation about the continuum limit of QCD.

\footnotetext{
${ }^{1}$ In the following we will use the notation $J^{P C}$ for the glueballs, where $J$ is the glueball spin, and $P, C$ refer to the parity and charge conjugation quantum numbers respectively.
} 
2. The corrections to the ratios of the glueball masses are relatively small compared to the correction to each glueball mass, suggesting that the corrections are somewhat universal for all the glueball masses. This may indicate that the good agreement between the supergravity computation and the lattice gauge theory results is not a coincidence but is due to small $\lambda^{-1}$ corrections to the mass ratios.

This paper is organized as follows.

In section 2 , we solve the supergravity wave equations in the $\mathrm{AdS}_{5}$ black hole geometry to obtain glueball masses in $\mathrm{QCD}_{3}$ and compare the results with lattice computations.

In section 's-i, we solve the supergravity wave equations in the $\mathrm{AdS}_{7}$ black hole geometry to obtain glueball masses in $\mathrm{QCD}_{4}$ and compare the results with lattice computations.

In section 'A action and to the $\mathrm{AdS}_{5}$ black hole geometry to estimate corrections to the glueball masses in $\mathrm{QCD}_{3}$.

We close the paper with a summary and discussions.

\section{Glueballs in three dimensions}

The $\mathcal{N}=4$ superconformal $S U(N)$ gauge theory in four dimensions is realized as a low energy effective theory of $N$ coinciding parallel D3 branes. One can construct a three-dimensional non-supersymmetric theory [i2] by compactifying this theory on $\mathbf{R}^{3} \times \mathbf{S}^{1}$ with anti-periodic boundary conditions on the fermions around the compactifying circle $\mathbf{S}^{1}$. Supersymmetry is broken explicitly by the boundary conditions. As the radius $R$ of the circle becomes small, the fermions decouple from the system since there are no zero frequency Matsubara modes. The scalar fields in the $4 \mathrm{D}$ theory will acquire masses at one-loop, since supersymmetry is broken, and these masses become infinite as $R \rightarrow 0$. Therefore in the infrared we are left with only the gauge field degrees of freedom and the theory should be effectively the same as pure $\mathrm{QCD}_{3}$.

According to Maldacena [i] IIB superstring theory on $\mathrm{AdS}_{5} \times \mathbf{S}^{5}$ with the metric

$$
\frac{d s^{2}}{l_{s}^{2} \sqrt{4 \pi g_{s} N}}=\rho^{-2} d \rho^{2}+\rho^{2} \sum_{i=1}^{4} d x_{i}^{2}+d \Omega_{5}^{2},
$$

where $l_{s}$ is the string length related to the superstring tension, $g_{s}$ is the string coupling constant and $d \Omega_{5}$ is the line element on $\mathbf{S}^{5}$. The $x^{1,2,3,4}$ directions in $\operatorname{AdS}_{5}$ correspond to $\mathbb{R}^{4}$ where the gauge theory lives. The gauge coupling constant $g_{4}$ of the $4 \mathrm{D}$ theory is related to the string coupling constant $g_{s}$ as $g_{4}^{2}=g_{s}$. In the 't Hooft limit $(N \rightarrow \infty$ with $g_{4}^{2} N=g_{s} N$ fixed), the string coupling constant vanishes $g_{s} \rightarrow 0$. Therefore we 
can study the $4 \mathrm{D}$ theory using the first quantized string theory in the AdS space ( $\left.12 . \overline{1}_{1}^{1}\right)$. Moreover if $g_{s} N \gg 1$, the curvature of the AdS space is small and the string theory is approximated by classical supergravity.

Upon compactification on $\mathbf{S}^{1}$ and imposing the supersymmetry breaking boundary conditions, $\left(2 . \overline{1}_{1}\right)$ is replaced by the Euclidean black hole geometry [2]

$$
\frac{d s^{2}}{l_{s}^{2} \sqrt{4 \pi g_{s} N}}=\left(\rho^{2}-\frac{b^{4}}{\rho^{2}}\right)^{-1} d \rho^{2}+\left(\rho^{2}-\frac{b^{4}}{\rho^{2}}\right) d \tau^{2}+\rho^{2} \sum_{i=1}^{3} d x_{i}^{2}+d \Omega_{5}^{2},
$$

where $\tau$ parameterizes the compactifying circle and the $x^{1,2,3}$ direction corresponding to the $\mathbb{R}^{3}$ where $\mathrm{QCD}_{3}$ lives. The horizon of this geometry is located at $\rho=b$ with

$$
b=\frac{1}{2 R} .
$$

Once again, the supergravity approximation is applicable for $N \rightarrow \infty$ and $g_{s} N \gg 1$.

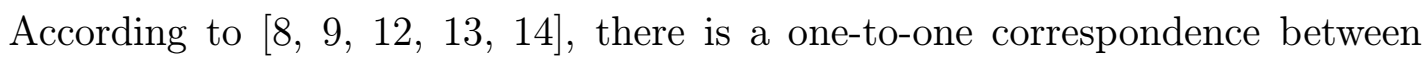
supergravity wave solutions on $\mathrm{AdS}_{5} \times \mathbf{S}^{5}$ and chiral primary fields (and their descendants) in the $\mathcal{N}=4$ superconformal theory in four dimensions. The mass $m$ of a $p$-form $C$ on the AdS space is related to the dimension $\Delta$ of a $(4-p)$ form operator in the $\mathcal{N}=4$ theory by

$$
m^{2}=(\Delta-p)(\Delta+p-4) .
$$

The supergravity fields on $\mathrm{AdS}_{5} \times \mathbf{S}^{5}$ can be classified by decomposing them into spherical harmonics (the Kaluza-Klein modes) on $\mathbf{S}^{5}$. They fall into irreducible representations of the $S O(6)$ isometry group of $\mathbf{S}^{5}$, which is also the $R$-symmetry group of the $4 \mathrm{D}$ superconformal theory. The spectrum of Kaluza-Klein harmonics of type

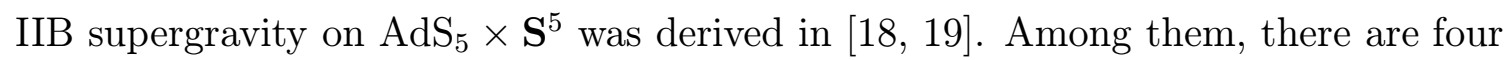
Kaluza-Klein modes that are $S O(6)$ singlets, coming from the $s$-wave components on $\mathbf{S}^{5}$ of bosonic fields. They are:

1. The graviton $g_{\mu \nu}$ polarized along the $\mathbb{R}^{4}$ in $\left({ }_{2}^{2} . \overline{2}_{1}^{1}\right)$. It couples to the dimension 4 stress-energy tensor $T_{\mu \nu}$ of the $\mathcal{N}=4$ theory.

2. The dilaton and the R-R scalar, which combine into a complex massless scalar field. Its real and imaginary parts couple to the dimension 4 scalar operators $\mathcal{O}_{4}=\operatorname{tr} F^{2}$ and $\tilde{\mathcal{O}}_{4}=\operatorname{tr} F \wedge F$ of the $\mathcal{N}=4$ theory respectively.

3. The NS-NS and R-R two-forms, which combine into a complex-valued antisymmetric field $A_{\mu \nu}$, polarized along the $\mathbb{R}^{4}$. Its (AdS mass) $)^{2}=16$ and using (2.4) we see that it couples to a dimension 6 two-form operator of the $\mathcal{N}=4$ theory. This operator has been identified as $\mathcal{O}_{6}=d^{a b c} F_{\mu \alpha}^{a} F^{b \alpha \beta} F_{\beta \nu}^{c}[\overline{2} \overline{2}, 12 \overline{3} \bar{i}$.

4. The $s$-wave component of the metric $g_{\alpha}^{\alpha}$ and the R-R 4-form $A_{\alpha \beta \gamma \delta}$ polarized along $\mathbf{S}^{5}$. They combine into a massive scalar with (AdS mass) $)^{2}=32$ and couple to a dimension 8 scalar operator constructed from the gauge field strength $F_{\mu \nu}$ of the $\mathcal{N}=4$ theory [2]年, $23 \overline{3}$. 
Only these $S O(6)$ singlet fields are related to glueballs of $\mathrm{QCD}_{3}$ since $S O(6)$ non-singlets are supposed to decouple in the limit $R \rightarrow 0$.

Let us discuss now how to identify the quantum numbers of the glueballs. The spin and the parity of a glueball in three dimensions can be easily found from the transformation properties of the corresponding supergravity field. The charge conjugation, $C$, for gluons is defined by $A_{\mu}^{a} T_{i j}^{a} \rightarrow-A_{\mu}^{a} T_{j i}^{a}$ where the $T^{a}$ 's are the hermitian generators of the gauge group [25]. In the string theory, charge conjugation corresponds to the worldsheet parity transformation changing the orientation of the open string attached to D-branes. Therefore, for example, the NS-NS two-form in supergravity is odd under the charge conjugation. This is consistent with the fact that it couples to $\mathcal{O}_{6}$, which indeed has $C=-1$.

From the point of view of $\mathrm{QCD}_{3}$, the radius $R$ of the compactifying circle provides the ultraviolet cutoff scale. To obtain large $N \mathrm{QCD}_{3}$ in the continuum, one has to take $g_{4}^{2} N \rightarrow 0$ as $R \rightarrow 0$ so that $g_{3}^{2} N=g_{4}^{2} N / R$ remains at the intrinsic energy scale of $\mathrm{QCD}_{3}$. Here $g_{3}$ is the dimensionful gauge coupling of $\mathrm{QCD}_{3}$. This is the opposite of the limit that is required for the supergravity description to be valid. As we mentioned, the supergravity description is applicable for $g_{4}^{2} N \gg 1$. Therefore, with the currently available techniques, the Maldacena-Witten conjecture can only be used to study large $N$ QCD with a fixed ultraviolet cutoff $R^{-1}$ in the strong ultraviolet coupling regime. The results we find are, however, surprisingly close to those of the lattice computation, leading us to suspect that $\left(g_{4}^{2} N\right)^{-1}$ corrections to the mass ratios are small. In section 恿, we will estimate the leading $\left(g_{4}^{2} N\right)^{-1}$ correction to our computation.

Consider first the $0^{++}$glueball masses. These can be derived from the 2-point function of the operator $\operatorname{tr} F_{\mu \nu} F^{\mu \nu}$. In the supergravity description we have to solve the classical equation of motion of the massless dilaton,

$$
\partial_{\mu}\left[\sqrt{g} \partial_{\nu} \Phi g^{\mu \nu}\right]=0,
$$

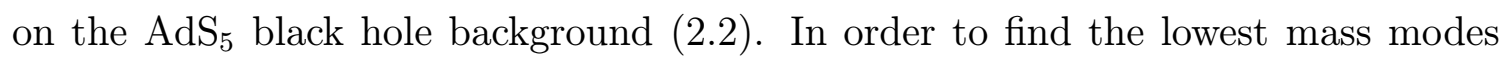
we assume following [2] that $\Phi$ is independent of $\tau$ and has the form $\Phi=f(\rho) e^{i k x}$. Using the metric of (2.2.

$$
\rho^{-1} \frac{d}{d \rho}\left(\left(\rho^{4}-b^{4}\right) \rho \frac{d f}{d \rho}\right)-k^{2} f=0 .
$$

Since the glueball mass $M^{2}$ is equal to $-k^{2}$, the task is to solve this equation as an eigenvalue problem for $k^{2}$. In the following we set $b=1$, so the masses are computed in units of $b$. If one changes variables to $x=\rho^{2}$, the equation takes the form,

$$
\frac{d^{2} f}{d x^{2}}+\left(\frac{1}{x}+\frac{1}{x-1}+\frac{1}{x+1}\right) \frac{d f}{d x}-\frac{k^{2}}{4 x\left(x^{2}-1\right)} f=0
$$

namely it is an ordinary differential equation with four regular singularities at $x=$ $0, \pm 1$ and $\infty$. 
Unlike the equation with three regular singularities (known as the hypergeometric equation), analytic solutions are not known for this type of equation. Fortunately there is an analytical method to compute its eigenvalues $k^{2}$. It is the exact WKB analysis recently developed by mathematicians at RIMS, Kyoto University [2] $\left.\overline{2}_{-}\right]$. To use their approach, we note that the differential equation (12.7. Schrödinger-type equation

$$
\left(-\frac{d^{2}}{d x^{2}}+Q(x)\right) g(x)=0
$$

where $g(x)=\sqrt{x\left(x^{2}-1\right)} f(x)$ and

$$
Q(x)=\frac{3 x^{4}-6 x^{2}-1}{4 x^{2}\left(x^{2}-1\right)^{2}}+\frac{k^{2}}{4 x\left(x^{2}-1\right)} .
$$

To apply the WKB analysis, one can perturb the equation as

$$
\left(-\frac{d^{2}}{d x^{2}}+Q(x)+\left(\eta^{2}-1\right) R(x)\right) g(x)=0,
$$

by introducing a large parameter $\eta$. With a suitable choice of $R(x)$, the secular equation, which determines the values of $k^{2}$ so that the equation admits a solution regular at both $x=1$ and $\infty$, becomes explicitly solvable as a asymptotic power series expansion in $\eta^{-1}$. Assuming the expansion is Borel summable at $\eta=1$, the eigenvalues are approximated by the following expression [2]

$$
k^{2}=-6 n(n+1), \quad(n=1,2,3, \ldots) .
$$

We should note that the differential equation in question is degenerate from the point of view of the exact WKB analysis and a mathematical proof of the Borel summability in this case has not been given. It is possible that the formula $(\overline{2}-11)$ receives small corrections.

Since the analytical expression ( $\left(2 . \overline{1}_{1} \overline{1}_{1}\right)$ for $k^{2}$ is still preliminary and we would like to find masses for the other glueball states, we also solved the differential equation $\left(\underline{2} . \overline{6}_{1}\right)$ numerically. For large $\rho$, the black hole metric $\left({ }^{2} .2_{2}^{\prime}\right)$ asymptotically approaches the AdS metric, and the behavior of the solution for a $p$-form for large $\rho$ takes the form $\rho^{\lambda}$, where $\lambda$ is determined from the mass $m$ of the supergravity field:

$$
m^{2}=\lambda(\lambda+4-2 p)
$$

Indeed both ( $\left(2 . \overline{6}_{1}^{\prime}\right)$ and $\left(\overline{2} . \overline{1} \overline{2}^{\prime}\right)$ give the asymptotic forms $f \sim 1, \rho^{-4}$, and only the later is a normalizable solution [2i]. Changing variables to $f=\psi / \rho^{4}$ we have:

$$
\left(\rho^{2}-\rho^{6}\right) \psi^{\prime \prime}+\left(3 \rho^{5}-7 \rho\right) \psi^{\prime}+\left(16+k^{2} \rho^{2}\right) \psi=0 .
$$


For large $\rho$ this equation can be solved by series solution with negative even powers:

$$
\psi=\Sigma_{n=0}^{\infty} a_{2 n} \rho^{-2 n} .
$$

Since the normalization is arbitrary we can set $a_{0}=1$. The first few coefficients are given by:

$$
\begin{aligned}
& a_{2}=\frac{k^{2}}{12}, \\
& a_{4}=\frac{1}{2}+\frac{k^{4}}{384}, \\
& a_{6}=\frac{7 k^{2}}{120}+\frac{k^{6}}{23040} .
\end{aligned}
$$

For $n \geq 5$ the coefficients are given by the recursive relation:

$$
\left(n^{2}+4 n\right) a_{n}=k^{2} a_{n-2}+n^{2} a_{n-4} .
$$

Since the black hole geometry is regular at the horizon $\rho=1, k^{2}$ has to be adjusted so that $f$ is also regular at $\rho=1[2]{ }^{2}$. This can be done numerically in a simple fashion using a "shooting" technique as follows. For a given value of $k^{2}$ the equation is numerically integrated from some sufficiently large value of $\rho\left(\rho \gg k^{2}\right)$ by matching $f(\rho)$ with the asymptotic solution set by $(2.141)$ and $\left(2.15 \overline{1}_{1}\right)$. The glueball mass $M$ is related to the eigenvalues of $k^{2}$ by $M^{2}=-k^{2}$ in units of $b^{2}$. The results of the numerical work are listed in table $\underset{1}{1}$. They agree with the formula (2.1 $\left.\overline{1}_{1}^{\prime}\right)$. The $4 \%$ discrepancy of the two results are either due to some systematic error in the numerical

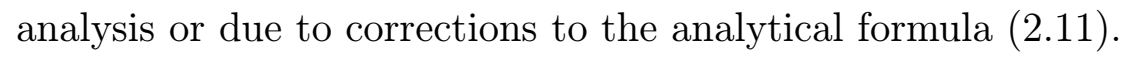

\begin{tabular}{|l|ccc|}
\hline state & numerical method & exact WKB method & ratio \\
\hline $0^{++}$ & 11.59 & 12 & 1.03 \\
$0^{++*}$ & 34.53 & 36 & 1.04 \\
$0^{++* *}$ & 68.98 & 72 & 1.04 \\
$0^{++* * *}$ & 114.9 & 120 & 1.04 \\
$0^{++* * * *}$ & 172.3 & 180 & 1.04 \\
$0^{++* * * * *}$ & 241.2 & 252 & 1.04 \\
\hline
\end{tabular}

Table 1: (Mass) $)^{2}$ of $0^{++}$glueball in $\mathrm{QCD}_{3}$ obtained by solving the supergravity wave equation in the black hole geometry (in units of $b^{2}$ ) using the two different methods.

Since both methods give the same results within a $4 \%$ error, we are ready to compare them with the lattice gauge theory computations [2]7. Since the lattice results are in units of string tension, we normalize the supergravity results so that the lightest $0^{++}$state agrees with the lattice result. The results are listed in table 2 One should also expect a systematic error in addition to the statistical error denoted in table $:_{-1}^{12}$ for the lattice computations.

\footnotetext{
${ }^{2}$ We thank A. Jevicki and J. P. Nunes for communication on the boundary condition.
} 


\begin{tabular}{|l|ccc|}
\hline state & lattice, $N=3$ & lattice, $N \rightarrow \infty$ & supergravity \\
\hline $0^{++}$ & $4.329 \pm 0.041$ & $4.065 \pm 0.055$ & 4.07 (input) \\
$0^{++*}$ & $6.52 \pm 0.09$ & $6.18 \pm 0.13$ & 7.02 \\
$0^{++* *}$ & $8.23 \pm 0.17$ & $7.99 \pm 0.22$ & 9.92 \\
$0^{++* * *}$ & - & - & 12.80 \\
$0^{++* * * *}$ & - & - & 15.67 \\
$0^{++* * * *}$ & - & - & 18.54 \\
\hline
\end{tabular}

Table 2: $0^{++}$glueball masses in $\mathrm{QCD}_{3}$ coupled to $\operatorname{tr} F_{\mu \nu} F^{\mu \nu}$. The lattice results are in units of the square root of the string tension. The denoted error in the lattice results is only the statistical one.

Next we consider the two-form of the supergravity theory. As noted previously, it couples to the operator $\mathcal{O}_{6}$. This operator contains $1^{+-}$and $1^{--}$components, which correspond to the fields $A_{\tau i}$ and $A_{i j}$, where $i, j=1,2,3$ correspond to the three coordinates $x_{i}$ of $\mathbb{R}^{3}$. The remaining components $A_{\rho \tau}$ and $A_{\rho i}$ can be set to zero by an appropriate gauge transformation. In the $\mathrm{QCD}_{3}$ limit $R \rightarrow 0$, the $1^{--}$ component $A_{i j}$ is reduced to a $0^{--}$operator in $3 \mathrm{D}$, and thus has a non-zero overlap with the $0^{--}$glueball. ${ }^{3}$ On the other hand, the $1^{+-}$components $A_{\tau i}$ couple to an operator which is supposed to decouple in the $R \rightarrow 0$ limit. Therefore they do not correspond to glueball states in $\mathrm{QCD}_{3}$.

The $s$-wave component of the two-form field satisfies the equation $[1] \overline{8}, 1,26]$

$$
\frac{3}{\sqrt{g}} \partial_{\mu}\left[\sqrt{g} \partial_{\left[\mu^{\prime}\right.} A_{\left.\mu_{1}^{\prime} \mu_{2}^{\prime}\right]} g^{\mu^{\prime} \mu} g^{\mu_{1}^{\prime} \mu_{1}} g^{\mu_{2}^{\prime} \mu_{2}}\right]-16 g^{\mu_{1}^{\prime} \mu_{1}} g^{\mu_{2}^{\prime} \mu_{2}} A_{\mu_{1}^{\prime} \mu_{2}^{\prime}}=0
$$

where [ ] denotes antisymmetrization with strength one. As before we look for solutions which are independent of $\tau$ and are of the form $A_{i j}=h_{i j}(\rho) e^{i k x}$. The $\rho \tau$ and the $\rho i$ components of this equation simply result in a constraint which sets the transverse component of $A_{i j}$ to zero. For the remaining pseudoscalar component from $A_{i j}$ the equation reduces to

$$
\rho\left(\rho^{4}-1\right) h^{\prime \prime}+\left(3+\rho^{4}\right) h^{\prime}-\left(k^{2} \rho+16 \rho^{3}\right) h=0,
$$

in units where $b=1$. At large $\rho$ the solution has the form

$$
h=\rho^{-4} \Sigma_{n=0}^{\infty} a_{2 n} \rho^{-2 n} .
$$

Since the normalization is arbitrary we can again set $a_{0}=1$. The first few coefficients are given by:

$$
a_{2}=\frac{k^{2}}{20}, \quad a_{4}=\frac{640+k^{4}}{960}, \quad a_{6}=\frac{3520 k^{2}+k^{6}}{80640} .
$$

\footnotetext{
${ }^{3}$ The parity $P=-1$ is due to the fact that the 2 -form is dual to a pseudoscalar. The charge conjugation $C=-1$ is inferred from the string worldsheet parity.
} 


\begin{tabular}{|l|ccc|}
\hline state & lattice, $N=3$ & lattice, $N \rightarrow \infty$ & supergravity \\
\hline $0^{--}$ & $6.48 \pm 0.09$ & $5.91 \pm 0.25$ & 6.10 \\
$0^{--*}$ & $8.15 \pm 0.16$ & $7.63 \pm 0.37$ & 9.34 \\
$0^{--* *}$ & $9.81 \pm 0.26$ & $8.96 \pm 0.65$ & 12.37 \\
$0^{--* * *}$ & - & - & 15.33 \\
$0^{--* * * *}$ & - & - & 18.26 \\
$0^{--* * * *}$ & - & - & 21.16 \\
\hline
\end{tabular}

Table 3: $0^{--}$glueball masses in $\mathrm{QCD}_{3}$ coupled to $\mathcal{O}_{6}$. The lattice results are in units of square root of the string tension. The normalization of the supergravity results is the same as in table

We have solved the differential equation and obtained the eigenvalues $k^{2}$ by the same numerical method described above. The results are shown in table is. The supergravity results are displayed in the same normalization as the one used in table 2

Since the supergravity method and the lattice gauge theory compute the glueball masses in different units, one cannot compare the absolute values of the lowest glueball mass obtained using these methods. However it makes sense to compare the lowest glueball masses of different quantum numbers. Using tables ${ }_{2}^{1}$, and $\hat{\beta}_{-1}$, we find that the supergravity results are in good agreement with the lattice gauge theory computation [2]ㄱㅁ:

$$
\left(\frac{M_{0^{--}}}{M_{0^{++}}}\right)_{\text {supergravity }}=1.50, \quad\left(\frac{M_{0^{--}}}{M_{0^{++}}}\right)_{\text {lattice }}=1.45 \pm 0.08 .
$$

There are still two more $S O(6)$ singlet supergravity fields. One of them is the $s$-wave component of the metric $g_{\alpha}^{\alpha}$ and the R-R 4-form $A_{\alpha \beta \gamma \delta}$ polarized along $\mathbf{S}^{5}$. From (12.4) we see that it should couple to a dimension 8 scalar operator $\mathcal{O}_{8}$. In $[\overline{2} \overline{2} \overline{4}, \overline{2} \overline{4}]$, this operator is identified as a symmetrized form of $\left[F^{4}-\frac{1}{4}\left(F^{2}\right)^{2}\right]$. By using the prescription of Tseytlin [20] to symmetrize the group indices, one finds that the operator is even under the charge conjugation. This is also seen from the fact that $g_{\alpha}^{\alpha}$ is clearly even both spacetime and worldsheet parity transformations. Therefore $g_{\alpha}^{\alpha}$ has the quantum numbers of the $0^{++}$glueball. The classical equation of motion of $g_{\alpha}^{\alpha}$ is that of a massive scalar with (AdS mass $)^{2}=32$ (in units of $b^{2}$ ) [i]

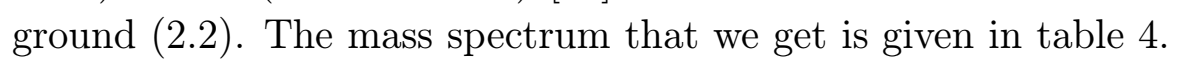

\begin{tabular}{|c|}
\hline$g_{\alpha}^{\alpha}$ and $A_{\alpha \beta \gamma \delta}$ \\
\hline 8.85 \\
12.06 \\
15.00 \\
17.98 \\
\hline
\end{tabular}

Table 4: $0^{++}$ glueball masses in $\mathrm{QCD}_{3}$ coupled to $\mathcal{O}_{8}$, the normalization is the same as in table $\overline{2}$. In the $g_{s} N \rightarrow \infty$ limit the operators $\mathcal{O}_{8}$ and $\operatorname{tr} F^{2}$ are not mixed since they couple to different states in the supergravity theory. However, we expect that for finite $g_{s} N$ these operators will mix, thus the full $0^{++}$spectrum is expected to be given by the interleaving of tables ${ }_{-1}^{-}$and ${ }_{-}^{1}$. For example the $0^{++* *}$ presumably corresponds to the first state in table , $_{-}^{\overline{1}}$. 
The remaining $S O(6)$ singlet is the graviton $g_{\mu \nu}$. It couples to the energymomentum tensor $T_{\mu \nu}$ and therefore corresponds to the $2^{++}$glueball. It would be interesting to compute its mass and compare with the lattice result.

\section{Glueballs in four dimensions}

To construct $\mathrm{QCD}_{4}$, one starts with the superconformal theory in six dimensions realized on $N$ parallel coinciding M5-branes. The compactification of this theory on a circle of radius $R_{1}$ gives a five-dimensional theory whose low-energy effective theory is the maximally supersymmetric $S U(N)$ gauge theory with gauge coupling constant $g_{5}^{2}=R_{1}$. To obtain $\mathrm{QCD}_{4}$, one compactifies this theory further on another $\mathbf{S}^{1}$ of radius $R_{2}$. The gauge coupling constant $g_{4}$ in $4 \mathrm{D}$ is given by $g_{4}^{2}=g_{5}^{2} / R_{2}=R_{1} / R_{2}$. To break supersymmetry, one imposes the anti-periodic boundary condition on the fermions around the second $\mathbf{S}^{1}$.

According to Maldacena [ii], the large $N$ limit of the six-dimensional theory is $M$ theory on $\mathrm{AdS}_{7} \times \mathbf{S}^{4}$. Upon compactification on $\mathbf{S}^{1} \times \mathbf{S}^{1}$ and imposing the antiperiodic boundary conditions around the second $\mathbf{S}^{1}$, we find $M$ theory to be on the black hole geometry [2]. To take the large $N$ limit while keeping $g_{4}^{2} N$ finite, we have to take $R_{1} \ll R_{2}$. In this limit, $M$ theory reduces to type IIA string theory and the M5 brane wrapping on $\mathbf{S}^{1}$ of radius $R_{1}$ becomes a $\mathrm{D} 4$ brane. The large $N$ limit of $\mathrm{QCD}_{4}$ then becomes string theory on the black hole geometry given by

$$
\frac{d s^{2}}{l_{s}^{2} g_{5}^{2} N / 4 \pi}=\frac{d \rho^{2}}{4 \rho^{3 / 2}\left(1-\frac{b^{6}}{\rho^{3}}\right)}+\rho^{3 / 2}\left(1-\frac{b^{6}}{\rho^{3}}\right) d \tau^{2}+\rho^{3 / 2} \sum_{i=1}^{4} d x_{i}^{2}+\rho^{1 / 2} d \Omega_{4}^{2},
$$

with a dilaton $e^{\phi} \sim \rho^{3 / 4}[\overline{2} \overline{2} \overline{9}]$. The location of the horizon $\rho=b^{2}$ is related to the radius $R_{2}$ of the compactifying circle as

$$
b=\frac{1}{3 R_{2}} .
$$

As in the case of three dimensions, we will compute the spectrum of glueball masses by solving the classical equations of motions of Kaluza-Klein modes of the supergravity theory. We will consider only singlets of the $S O(5)$ isometry group of $\mathbf{S}^{4}$, which corresponds to the $R$-symmetry group of the six-dimensional theory.

Consider first the $0^{++}$glueball. The non-extremal D4 brane solution has a non

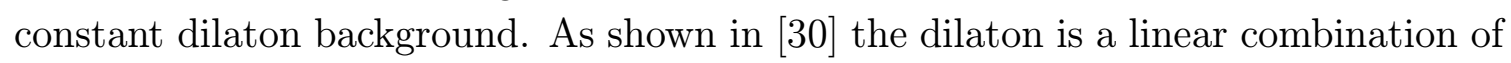
two scalars. One of them is massless and couples to the relevant glueball operator. The equation of motion for the scalar is given by (2. $\left.2_{1}\right)$ in the background of the metric $\left(\overline{1}_{1}\right)$. Again assuming that the solution is independent of $\tau$ and of the form $\Phi=f(\lambda) e^{i k x}$ (with $\lambda^{2}=\rho$ ), one obtains the differential equation in the units where $b=1$ as

$$
\left(\lambda^{7}-\lambda\right) f^{\prime \prime}(\lambda)+\left(10 \lambda^{6}-4\right) f^{\prime}(\lambda)-\lambda^{3} k^{2} f(\lambda)=0
$$


The asymptotic solutions to this equation are $f \sim 1, \lambda^{-9}$, with the latter corresponding to normalizable solutions. In order to solve the equation and find the allowed values of $k^{2}$ we introduce the function $g(\lambda)$ as $f(\lambda)=\lambda^{-9} g(\lambda)$. This way $g(\lambda)$ has to be asymptotically constant for $\lambda \rightarrow \infty$, and one can again look for a solution in terms of a negative power series in $\lambda$. The differential equation for $g(\lambda)$ is

$$
\left(\lambda^{8}-\lambda^{2}\right) g^{\prime \prime}+\left(14 \lambda-8 \lambda^{7}\right) g^{\prime}-\left(\lambda^{4} k^{2}+54\right) g=0 .
$$

The first few coefficients in the power series solution $g=\sum_{n=0}^{\infty} a_{2 n} \lambda^{-2 n}$ are given by (for $a_{0}=1$ )

$$
a_{2}=\frac{k^{2}}{22}, \quad a_{4}=\frac{k^{4}}{1144}, \quad a_{6}=\frac{61776+k^{6}}{102960} .
$$

The regularity of $f$ at $\lambda=1$, after numerically solving the equation (……4i $)$ as described in the previous section, results in the allowed values of $k^{2}$. The first six masses (normalized so that the lightest $0^{++}$state agrees with the lattice calculation) together with the available lattice results $[32,3,3]$ are given in table $\overline{1}$.

In order to calculate the masses of the $0^{-+}$glueball in four dimensions we will consider the 3 -form $A_{\alpha \beta \gamma}$ of the eleven dimensional supergravity. In this case, it is more useful to use the elevendimensional metric

\begin{tabular}{|l|cc|}
\hline state & lattice, $N=3$ & supergravity \\
\hline $0^{++}$ & $1.61 \pm 0.15$ & 1.61 (input) \\
$0^{++*}$ & 2.8 & 2.38 \\
$0^{++* *}$ & - & 3.11 \\
$0^{++* * *}$ & - & 3.82 \\
$0^{++* * * *}$ & - & 4.52 \\
$0^{++* * * * *}$ & - & 5.21 \\
\hline
\end{tabular}

Table 5: Masses of the first few $0^{++}$glueballs in $\mathrm{QCD}_{4}$, in $\mathrm{GeV}$, from supergravity compared to the available lattice results. Note that the authors of ref. [3īinj] do not quote errors for the $0^{++*}$ since it is not yet clear whether it is a genuine excited state or merely a two glueball bound state.

$$
d s^{2}=\frac{d \lambda^{2}}{\left(\frac{\lambda^{2}}{b^{2}}-\frac{b^{4}}{\lambda^{4}}\right)}+\left(\frac{\lambda^{2}}{b^{2}}-\frac{b^{4}}{\lambda^{4}}\right) d \tau^{2}+\lambda^{2} \sum_{i=1}^{5} d x_{i}^{2}+d \Omega_{4}^{2},
$$

which reduces to (3.1) upon compactifying $x_{5}$ on $\mathbf{S}^{1}$ and by going to the string frame [2]ㄹ] by multiplying the metric by $\lambda$, setting $\lambda^{2}=\rho$, and rescaling the other coordinates. The $s$-wave component of the 3 -form in the harmonic expansion on $\mathbf{S}^{4}$ is a singlet of

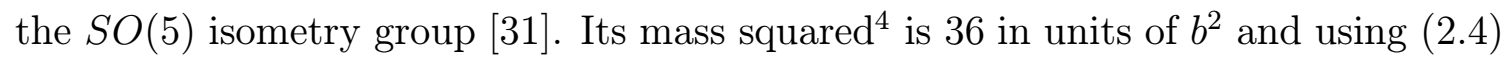
we see that it couples to a dimension 9 operator of the six-dimensional theory. The 3 -form obeys the following equation of motion:

$$
\frac{4}{\sqrt{g}} \partial_{\mu}\left[\sqrt{g} \partial_{\left[\mu^{\prime}\right.} A_{\left.\mu_{1}^{\prime} \mu_{2}^{\prime} \mu_{3}^{\prime}\right]} g^{\mu^{\prime} \mu} g^{\mu_{1}^{\prime} \mu_{1}} g^{\mu_{2}^{\prime} \mu_{2}} g^{\mu_{3}^{\prime} \mu_{3}}\right]-36 g^{\mu_{1}^{\prime} \mu_{1}} g^{\mu_{2}^{\prime} \mu_{2}} g^{\mu_{3}^{\prime} \mu_{3}} A_{\mu_{1}^{\prime} \mu_{2}^{\prime} \mu_{3}^{\prime}}=0 .
$$

\footnotetext{
${ }^{4}$ The value of the mass term is fixed by matching the supergravity computation [3] [3]
} 
Choosing a gauge where $A_{\rho \tau i}$ and $A_{\rho i j}$ vanish, where $i, j=1, \ldots, 5$, and assuming that the remaining components are independent of the coordinate $\tau$ and the only dependence on $x_{i}$ is through $e^{i k x}$, one finds that there are two independent modes after compactification to 4D:

1. A three-index tensor $A_{i j k}$. This is dual to a massive scalar and can be identified with the $0^{-+}$glueball of the $4 \mathrm{D}$ theory.

2. A massive vector $A_{\tau i j}$. This couples to an operator which is supposed to decouple in the limit $R_{2} \rightarrow 0$. Therefore it does not correspond to a glueball state in $\mathrm{QCD}_{4}$.

The scalar component of $A_{i j k}$ satisfies the differential equation

$$
\left(\lambda^{7}-\lambda\right) f^{\prime \prime}(\lambda)+\left(\lambda^{6}+5\right) f^{\prime}(\lambda)-\lambda^{3}\left(k^{2}+36 \lambda^{2}\right) f(\lambda)=0
$$

in the same units as in the equation (3.3) for the dilaton. The normalizable asymptotic solution behaves like $1 / \lambda^{6}$, thus we introduce the function $g(\lambda)$ by $f(\lambda)=$ $\lambda^{-6} g(\lambda)$. This satisfies

$$
\left(\lambda^{7}-\lambda\right) g^{\prime \prime}(\lambda)-\left(11 \lambda^{6}-17\right) g^{\prime}(\lambda)-\left(72+k^{2} \lambda^{4}\right) g(\lambda)=0 .
$$

The power series expansion $g(\lambda)=\sum_{n=0}^{\infty} a_{2 n} \lambda^{-2 n}$ with $a_{0}=1$ has the first few coefficients

$$
a_{2}=\frac{k^{2}}{28}, \quad a_{4}=\frac{k^{4}}{1792}, \quad a_{6}=\frac{129024+k^{6}}{193536} .
$$

The single-valuedness of the solution requires $g^{\prime}=6 g$ at $\lambda=1$. With this boundary condition, the numerical solution of ( $\left(\bar{y}_{1}^{\prime}\right)$ yields the allowed values of $k^{2}$, and the corresponding masses in the units defined above are displayed in table $\overline{\underline{6}}$.

Unlike the 3D case, there exists little lattice data on the masses of the excited glueball states. We can however compare the ratio of masses of the low-

\begin{tabular}{|l|cc|}
\hline state & lattice, $N=3$ & supergravity \\
\hline $0^{-+}$ & $2.19 \pm 0.32$ & 1.83 \\
$0^{-+*}$ & - & 2.67 \\
$0^{-+* *}$ & - & 3.42 \\
$0^{-+* * *}$ & - & 4.14 \\
$0^{-+* * * *}$ & - & 4.85 \\
$0^{-+* * * * *}$ & - & 5.55 \\
\hline
\end{tabular}
est glueball states $0^{-+}$and $0^{++}$

$$
\left(\frac{M_{0^{-+}}}{M_{0^{++}}}\right)_{\text {supergravity }}=1.14 \quad\left(\frac{M_{0^{-+}}}{M_{0^{++}}}\right)_{\text {lattice }}=1.36 \pm 0.32,
$$

and the results are in agreement within the one $\sigma$ error. 


\section{Leading string theory corrections}

As we mentioned earlier, the supergravity computation is valid in the strong ultraviolet coupling limit $g_{s} N \gg 1$. In order to compare with the lattice computations in the continuum limit, we have to take $g_{s} N \rightarrow 0$ as we take the ultraviolet cutoff $R^{-1} \rightarrow \infty$ so that the scale set by the Yang-Mills coupling constant remains at the intrinsic energy scale of QCD. The fact that the glueball masses computed in the supergravity limit are in good agreement with the lattice results leads us to suspect that, for this particular computation, $\alpha^{\prime}$ corrections are small. In this section, we test this idea.

For $g_{s} N \ll 1$, the curvature of the black hole geometry becomes larger than the string scale. Therefore stringy corrections (to be precise, the worldsheet sigma-model corrections) are expected to become important. The leading stringy corrections to the low-energy supergravity action were obtained in [i] $15,1,16]$. Recently Gubser, Klebanov and Tseytlin [i] corrections to the black hole metric. We use their result to calculate the leading corrections to the glueball mass spectrum. We will perform this computation only for the $0^{++}$glueballs in $\mathrm{QCD}_{3}$. We expect, however, that the conclusions will be similar for the other glueball states.

According to [i]i], the leading (in units of the curvature) $\alpha^{\prime}=\left(4 \pi g_{s} N\right)^{-1 / 2}$ correction to the $\mathrm{AdS}_{5}$ black hole metric (2).2.

$$
\frac{d s^{2}}{l_{s}^{2} \sqrt{4 \pi g_{4}^{2} N}}=\left(1+\delta_{1}\right) \frac{d \rho^{2}}{\left(\rho^{2}-\frac{b^{4}}{\rho^{2}}\right)}+\left(1+\delta_{2}\right)\left(\rho^{2}-\frac{b^{4}}{\rho^{2}}\right) d \tau^{2}+\rho^{2} \sum_{i=1}^{3} d x_{i}^{2}
$$

where the correction terms $\delta_{1,2}$ are given by the formulae

$$
\delta_{1}=+15 \gamma\left(5 \frac{b^{4}}{\rho^{4}}+5 \frac{b^{8}}{\rho^{8}}-19 \frac{b^{12}}{\rho^{12}}\right), \quad \delta_{2}=-15 \gamma\left(5 \frac{b^{4}}{\rho^{4}}+5 \frac{b^{8}}{\rho^{8}}-3 \frac{b^{12}}{\rho^{12}}\right)
$$

and $\gamma$ is given by $\gamma=\frac{1}{8} \zeta(3) \alpha^{\prime 3}$. With these corrections of the metric, the dilaton is no longer constant, instead it is given by

$$
\Phi_{0}=-\frac{45}{8} \gamma\left(\frac{b^{4}}{\rho^{4}}+\frac{b^{8}}{2 \rho^{8}}+\frac{b^{12}}{3 \rho^{12}}\right)
$$

There is also a correction to the ten-dimensional dilaton action $\left[1 \overline{1}_{1}^{\prime}, 1\right]$

$$
I_{\text {dilaton }}=-\frac{1}{16 \pi G_{10}} \int d^{10} x \sqrt{g}\left[-\frac{1}{2} g^{\mu \nu} \partial_{\mu} \Phi \partial_{\nu} \Phi+\gamma e^{-\frac{3}{2} \Phi} W\right]
$$

where $W$ is given in terms of the Weyl tensor, and in our background $W=180 / \rho^{16}$ in units where $b=1$. To the leading order in $\gamma$, the dilaton perturbation does not mix with the metric perturbation, so we can study the dilaton equation derived from 
the action $(\bar{A} . \overline{4})$ in the fixed metric background ( $(\bar{A} . \overline{1})$. In subleading order in $\gamma$, the term $\gamma e^{-\frac{3}{2} \Phi} W$ would generate a mixing of the dilaton and the graviton.

We now have all pieces needed in order to obtain the first order correction to the

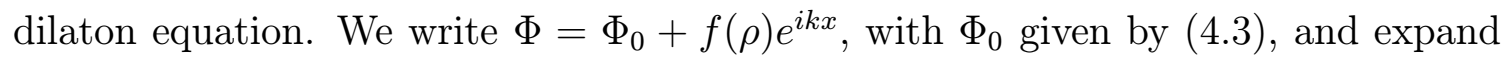
$f(\rho)$ and $k^{2}$ in $\gamma$ as

$$
f(\rho)=f_{0}(\rho)+\gamma h(\rho), \quad k^{2}=k_{0}^{2}+\gamma \delta k^{2} .
$$

Here $f_{0}(\rho)$ obeys the lowest order equation $\left({ }_{2}^{2} . \overline{6}_{1}^{\prime}\right)$ and is a numerically given function, and $k_{0}^{2}$ are the eigenvalues numerically obtained from the solution of $\left(\overline{1}_{2} . \overline{6}_{-}^{\prime}\right)$. The first order term of the differential equation obtained from the action ( $\left(\begin{array}{c}1 \\ .\end{array} \bar{A}_{1}^{\prime}\right)$ using the background $\left({ }_{-}^{\prime} \bar{A} \overline{1}_{1}^{\prime}\right)$ and $\left({ }_{-}^{\prime} \bar{A} . \overline{3}_{-}^{\prime}\right)$ is given by

$$
\begin{aligned}
\rho^{-1} \frac{d}{d \rho}\left(\left(\rho^{4}-1\right) \rho \frac{d h}{d \rho}\right)-k_{0}^{2} h= & \left(75-240 \rho^{-8}+165 \rho^{-12}\right) \frac{d^{2} f_{0}}{d \rho^{2}} \\
& +\left(75+1680 \rho^{-8}-1815 \rho^{-12}\right) \rho^{-1} \frac{d f_{0}}{d \rho} \\
& +\left(\delta k^{2}-120 k_{0}^{2} \rho^{-12}-405 \rho^{-14}\right) f_{0}(\rho) .
\end{aligned}
$$

With $f_{0}(\rho)$ and $k_{0}^{2}$ given, one may regard this as an inhomogeneous version of the equation ( $\left(\bar{b}_{2} \cdot \overline{6}_{1}\right)$. We solve this equation to determine $h(\rho)$ and $\delta k^{2}$.

At large $\rho$ the solution for the first order correction has the form

$$
h=\rho^{-4} \Sigma_{n=0}^{\infty} b_{2 n} \rho^{-2 n} .
$$

Since ("A. $\left.\bar{C}_{i}^{\prime}\right)$ is inhomogeneous for $h(\rho)$, one can always add to a solution $h(\rho)$ a constant multiple of the solution $f_{0}(\rho)$ of the corresponding homogeneous equation $\left(\underline{2} . \overline{6}_{1}^{\prime}\right)$. We use this freedom to set $b_{0}=0$. The first few coefficients are then given by:

$$
\begin{aligned}
& b_{2}=\frac{\delta k^{2}}{12}, \quad b_{4}=\frac{14400+2 \delta k^{2} k_{0}^{2}}{384}, \\
& b_{6}=\frac{1344 \delta k^{2}+100800 k_{0}^{2}+3 \delta k^{2} k_{0}^{4}}{23040} .
\end{aligned}
$$

\begin{tabular}{|l|ccc|}
\hline state & $\left(-k_{0}^{2}\right)$ & $\left(-\delta k^{2}\right)$ & $\delta k^{2} / k_{0}^{2}$ \\
\hline $0^{++}$ & 11.59 & 89.75 & 7.74 \\
$0^{++*}$ & 34.53 & 365.7 & 10.59 \\
$0^{++* *}$ & 68.98 & 809.8 & 11.74 \\
$0^{++* * *}$ & 114.9 & 1397 & 12.16 \\
$0^{++* * * *}$ & 172.3 & 2122 & 12.32 \\
$0^{++* * * * *}$ & 241.2 & 2991 & 12.40 \\
\hline
\end{tabular}

Table 7: Leading string correction to the $0^{++}$glueball masses in $\mathrm{QCD}_{3}$. The

We can now determine $\delta k^{2}$ by the same shooting method described above for each eigenvalue of $k_{0}^{2}$ and its corresponding eigenfunction $f_{0}(\rho)$. It turns out that, for each eigenvalue $k_{0}^{2}$, there is is a unique solution with $h$ first column gives the zeroth order supergravity result for the mass squared, the second column gives the coefficient of the leading string correction and the third column gives their ratio.

being regular at $\rho=1$. The first few solutions are shown in table 
Recalling that the squared mass of each glueball states is given by

$$
M^{2}=-\left(k_{0}^{2}+\frac{1}{8} \delta k^{2} \zeta(3) \alpha^{\prime 3}+\cdots\right) b^{2},
$$

we see that the leading stringy corrections to the $0^{++}$glueball masses are

$$
\begin{aligned}
M_{0^{++}}^{2} & =11.59 \times\left(1+0.97 \zeta(3) \alpha^{\prime 3}+\cdots\right) b^{2}, \\
M_{0^{++*}}^{2} & =34.53 \times\left(1+1.32 \zeta(3) \alpha^{\prime 3}+\cdots\right) b^{2}, \\
M_{0^{++* *}}^{2} & =68.98 \times\left(1+1.47 \zeta(3) \alpha^{\prime 3}+\cdots\right) b^{2}, \\
M_{0^{++* * *}}^{2} & =114.9 \times\left(1+1.52 \zeta(3) \alpha^{\prime 3}+\cdots\right) b^{2}, \\
M_{0^{++* * * *}}^{2} & =172.3 \times\left(1+1.54 \zeta(3) \alpha^{\prime 3}+\cdots\right) b^{2}, \\
M_{0^{++* * * *}}^{2} & =241.2 \times\left(1+1.55 \zeta(3) \alpha^{\prime 3}+\cdots\right) b^{2} .
\end{aligned}
$$

It is important to note that the relation between the radius $R$ of the compactifying circle $\mathbb{R}^{4} \rightarrow \mathbb{R}^{3} \times \mathbf{S}^{1}$ and the location $\rho=b$ of the horizon also receives an $\alpha^{\prime}$ -

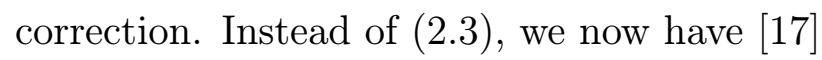

$$
b=\left(1-\frac{15}{8} \zeta(3) \alpha^{\prime 3}+\cdots\right) \frac{1}{2 R} .
$$

Therefore, in units of the ultraviolet cutoff $\Lambda_{U V}=(2 R)^{-1}$, the glueball masses are expressed as

$$
\begin{aligned}
M_{0^{++}}^{2} & =11.59 \times\left(1-2.78 \zeta(3) \alpha^{\prime 3}+\cdots\right) \Lambda_{U V}^{2}, \\
M_{0^{++*}}^{2} & =34.53 \times\left(1-2.43 \zeta(3) \alpha^{\prime 3}+\cdots\right) \Lambda_{U V}^{2}, \\
M_{0^{++* *}}^{2} & =68.98 \times\left(1-2.28 \zeta(3) \alpha^{\prime 3}+\cdots\right) \Lambda_{U V}^{2}, \\
M_{0^{++* * *}}^{2} & =114.9 \times\left(1-2.23 \zeta(3) \alpha^{\prime 3}+\cdots\right) \Lambda_{U V}^{2}, \\
M_{0^{++* * * *}}^{2} & =172.3 \times\left(1-2.21 \zeta(3) \alpha^{\prime 3}+\cdots\right) \Lambda_{U V}^{2}, \\
M_{0^{++* * * *}}^{2} & =241.2 \times\left(1-2.20 \zeta(3) \alpha^{\prime 3}+\cdots\right) \Lambda_{U V}^{2}
\end{aligned}
$$

Thus the glueball masses are indeed modified by the $\alpha^{\prime}=\left(4 \pi g_{s} N\right)^{-1 / 2}$ correction. The corrections are negative for all the 6 levels we computed and are of the order $\alpha^{\prime 3}$. Therefore the glueball masses decrease as we decrease the 't Hooft coupling $\lambda=g_{s} N$. As we discussed in the introduction of this paper, this is in accordance with our expectation about the continuum limit of QCD.

At the same time, the $\alpha^{\prime}$ corrections to the ratios of the masses appear to be smaller than the corrections to each glueball mass, suggesting that the corrections 
are somewhat universal. This may indicate that the good agreement between the supergravity computation and the lattice gauge theory results is not a coincidence but is due to small $\lambda^{-1}$ corrections to the mass ratios. Obviously, with the given data, we cannot tell whether the stringy corrections for the mass ratios remain small in the continuum limit $\lambda \rightarrow 0$. It would be very interesting to see whether this trend continues in the subleading corrections in $\alpha^{\prime}$.

\section{Summary and discussion}

In this paper, we computed the glueball masses of large $N$ QCD in three and four dimensions by solving supergravity wave equations in the AdS black hole geometry. The supergravity approximation is valid for large $N$ and large $\lambda=g_{Y M}^{2} N$ and therefore the results are to be compared with a fixed ultraviolet cutoff in the strong ultraviolet coupling regime.

We computed the ratios of the masses of the excited glueball states with the mass of the lowest state, as well as the ratio of masses of two different lowest glueball states. These ratios are in surprisingly good agreement with the available lattice data. We also computed the leading $\lambda^{-3 / 2}$ corrections to the glueball masses taking into account stringy corrections to the black hole geometry. We found that the corrections to the masses are in accordance with our expectation about the continuum limit of QCD. The corrections to the ratios of the masses appear to be smaller than the corrections to each glueball mass, suggesting that the corrections are somewhat universal.

The above computations can be generalized to higher spin glueballs. As noted previously, the graviton couples to the energy-momentum tensor and solving its equation of motion will give the masses of the $2^{++}$glueball. In general the higher spin glueballs will correspond to operators that couple to massive string excitations. The dimensions of these operators are $\Delta \sim \lambda^{1 / 4}$ for large $\lambda$ [隆]. It would be interesting to see how to extrapolate this result to the continuum $\lambda \rightarrow 0$.

Another interesting issue is the existence of $S O(6)$ non-singlet states in supergravity. For large $\lambda$, their masses are of the same order as the $S O(6)$ singlet states we studied in this paper. In the continuum limit, $\Lambda_{U V} \rightarrow \infty$ and $\lambda \rightarrow 0$, those states should decouple. Presumably $\lambda^{-1}$ corrections make them heavy.

Maldacena's conjecture reduces the problem of solving large $N$ QCD in three and four dimensions to that of controlling the $\alpha^{\prime}$ corrections to the two-dimensional sigmamodel with the Ramond-Ramond background. In this paper, we have extracted information about glueballs in strongly coupled QCD using the $\alpha^{\prime}$-expansion of the sigma-model. It would certainly be interesting to understand properties of such a sigma-model better. 


\section{Note added}

Recently the masses of the $S O(6)$ non-singlet states have been computed in [3-in] where it was found that they are comparable to those of the glueballs computed in this paper. It was also shown that the leading $\lambda^{-1}$ corrections computed using the metric (4.1) do not make these states heavier than the glueballs. Therefore, the decoupling of the $S O(6)$ non-singlet states is not evident to this order.

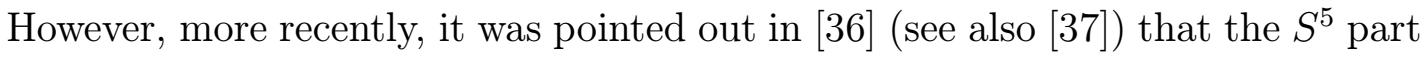
of the metric also receives the $O\left(\alpha^{\prime 3}\right)$ correction. The glueball masses computed in this paper would not be affected by such a correction since they correspond to states that we are constant on $S^{5}$. On the other hand, the masses of the $S O(6)$ non-singlet states would in general be sensitive to the correction of the $S^{5}$ part of the metric. In view of the importance of the issue, we revisited the computation of [355] and obtained the following result:

$$
\begin{aligned}
& M_{0}^{2}=11.59 \times\left(1-2.78 \zeta(3) \alpha^{\prime 3}+\cdots\right) \Lambda_{U V}^{2}, \\
& M_{1}^{2}=19.43 \times\left(1-2.73 \zeta(3) \alpha^{\prime 3}+\cdots\right) \Lambda_{U V}^{2}, \\
& M_{2}^{2}=29.26 \times\left(1-2.74 \zeta(3) \alpha^{\prime 3}+\cdots\right) \Lambda_{U V}^{2}, \\
& M_{3}^{2}=41.10 \times\left(1-2.78 \zeta(3) \alpha^{\prime 3}+\cdots\right) \Lambda_{U V}^{2}, \\
& M_{4}^{2}=54.93 \times\left(1-2.84 \zeta(3) \alpha^{\prime 3}+\cdots\right) \Lambda_{U V}^{2}, \\
& M_{5}^{2}=70.76 \times\left(1-2.89 \zeta(3) \alpha^{\prime 3}+\cdots\right) \Lambda_{U V}^{2}, \\
& M_{6}^{2}=88.60 \times\left(1-2.95 \zeta(3) \alpha^{\prime 3}+\cdots\right) \Lambda_{U V}^{2}, \\
& M_{7}^{2}=108.4 \times\left(1-3.01 \zeta(3) \alpha^{\prime 3}+\cdots\right) \Lambda_{U V}^{2} .
\end{aligned}
$$

This is to be compared with (3.9) of the paper [35. $M_{l}$ is the mass of the dilaton corresponding to the $l$-th Kaluza-Klein excitation on $S^{5}$. One finds that the correction to the metric (4.1) found in $[\underline{3} \overline{6} \overline{6}]$ does not significantly modify the

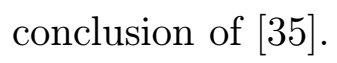

Therefore, even with the correction to the metric found in $[\underline{3} \overline{6} \overline{6}]$, the decoupling of the $S O(6)$ non-singlet states is not evident. It would be very interesting to understand whether higher order $\alpha^{\prime}$ corrections cause their decoupling and if so at what level (whether perturbatively or non-perturbatively in $\alpha^{\prime}$ ). This issue is closely related to the question of why the glueball masses computed in this paper is in such an excellent numerical agreement, and in general how well the string theory on the AdS Black Hole describes the non-supersymmetric Yang-Mills theory. 


\section{Acknowledgments}

We would like to thank M. Chanowitz, D. Gross, A. Hashimoto, C. Morningstar, S. Sharpe and Y. Takei for discussions and communications. We also thank Harlan Robins and Jonathan Tannenhauser, who participated in an early stage of this project, for discussions. H.O. and Y.O. thank the Institute for Theoretical Physics at Santa Barbara for its hospitality. This work was supported in part by the NSF grant PHY-95-14797 and in part the DOE grant DE-AC03-76SF00098. In addition, H.O. and Y.O. are supported by the NSF grant PHY-94-07194 through the ITP. C.C. is a research fellow of the Miller Institute for Basic Research in Science.

\section{References}

[1] J.M. Maldacena, The large $N$ limit of superconformal field theories and supergravity,

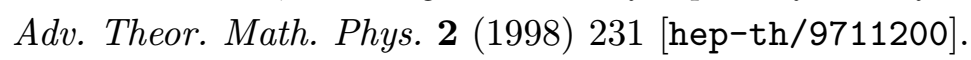

[2] E. Witten, Anti-de Sitter space, thermal phase transition, and confinement in gauge theories, iAdv. Theor Math Phys. 2 (1998) 5051 [Lep-th/9803131].

[3] A. Brandhuber, N. Itzhaki, J. Sonnenschein and S. Yankielowicz, Wilson loops in the

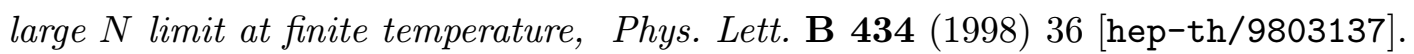

[4] S.-J. Rey, S. Theisen and J. Yee, Wilson-Polyakov loop at finite temperature in large

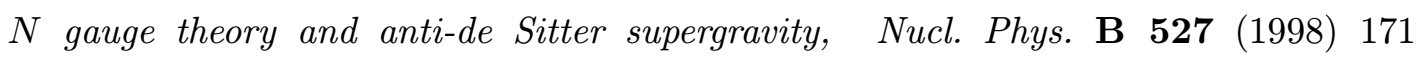
hep-th/9803135i.

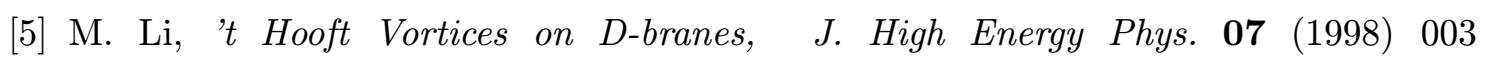
hep-th/9803252'; 't Hooft vortices and phases of large $N$ gauge theory,

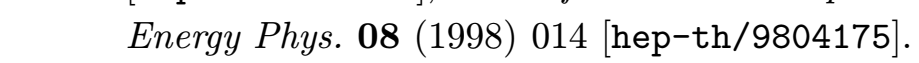

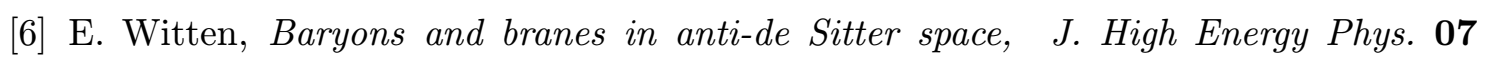
:

[7] D.J. Gross and H. Ooguri, Aspects of large $N$ gauge theory dynamics as seen by string

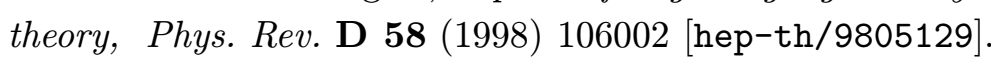

[8] S.S. Gubser, I.R. Klebanov, A.M. Polyakov, Gauge theory correlators from non-critical string theory, 'Ṕhys. Lett. B $\mathbf{4} 28$ (1998) 105' lihep-th/9802109'].

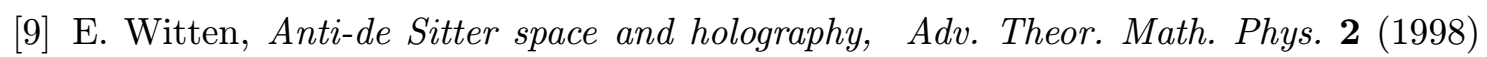

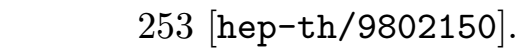

[10] I.R. Klebanov, World volume approach to absorption by non-dilatonic branes, iNucl. ( [hep-th/9703040i].

[11] S.S. Gubser, I.R. Klebanov and A.A. Tseytlin, String theory and classical absorption by threebranes, iNucl. Phys. B 499 11997$) 217$ [nep-th/9703040i]. 
[12] G. Horowitz and H. Ooguri, Spectrum of large $N$ gauge theory from supergravity, Phys. Rev. Lett. 80 (1998) 1116 hep-th/9802116i.

[13] S. Ferrara, C. Fronsdal and A. Zaffaroni, On $\mathcal{N}=8$ supergravity on $A d S_{5}$ and $\mathcal{N}=4$ superconformal Yang-Mills theory, 'Nucl.

[14] Y. Oz and J. Terning, Orbifolds of $A d S_{5} \times S^{5}$ and $4 d$ conformal field theories, iNucucl:

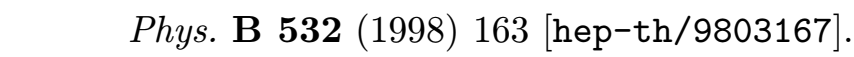

[15] M.T. Grisaru, A.E.M. van de Ven and D. Zanon, Four-loop beta function for the $\mathcal{N}=1$ and $\mathcal{N}=2$ supersymmetric nonlinear sigma-model in two dimensions, 'Phys. Lett. $\overline{\mathbf{B}}$ '

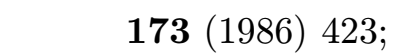

M. T. Grisaru and D. Zanon, Sigma-model superstring corrections to the Einstein-

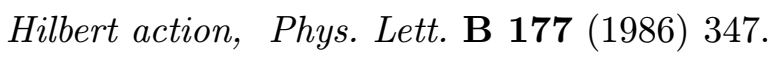

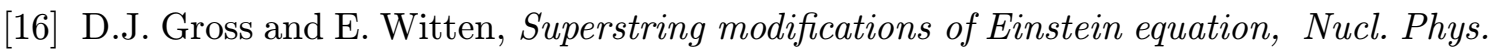
(1)

[17] S.S. Gubser, I.R. Klebanov and A.A. Tseytlin, Coupling constant dependence in the thermodynamics of $\mathcal{N}=4$ supersymmetric Yang-Mills theory, :

[18] H.J. Kim, L.J. Romans, P. Van Nieuwenhuizen, Mass spectrum of chiral ten-

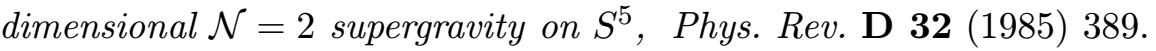

[19] M. Gunaydin, N. Marcus, The spectrum of the $S^{5}$ compactification of chiral $\mathcal{N}=$

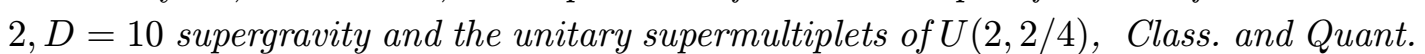
Grav. $2(1985) \bar{L} \overline{1} 1$

[20] T. Aoki, T. Kawai and Y. Takei, Algebraic analysis of singular perturbations - on exact WKB analysis, Sugaku Exposition 8-2 (1995) 217, and references therein.

[21] Y. Takei and T. Koike, private communication.

[22] S. Das and S. Trivedi, Three brane action and the correspondence between $\mathcal{N}=4$ Yang Mills theory and anti-de Sitter space, iphys. Lett. B 445 (1998) 1422] [hep-th/9804149].

[23] S. Ferrara, M. A. Lledo, A. Zaffaroni, Born-Infeld corrections to D3 brane action in

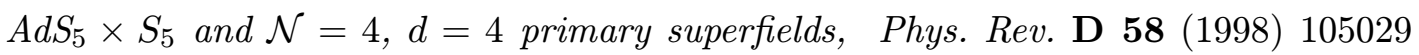
hep-th/9805082i].

[24] S.S. Gubser, A. Hashimoto, I.R. Klebanov, M. Krasnitz, Scalar absorption and the

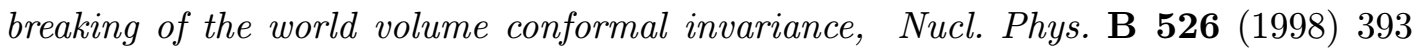
[hep-th/9803023il.

[25] J. Mandula, G. Zweig, and J. Govaerts, Covariant lattice glueball fields, iN Nucl. Phys.: (-

[26] S. Mathur and A. Matusis, Absorption of partial waves by three-branes, hep-th/9805064 
[27] M.J. Teper, $S U(N)$ gauge theories in $(2+1)$ dimensions, 'Pִ ;

[28] A.A. Tseytlin, On non-abelian generalization of Born-Infeld action in string theory,

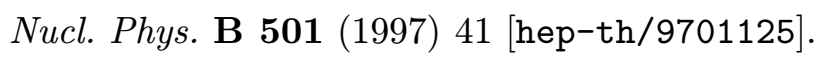

[29] N. Itzhaki, J. Maldacena, J. Sonnenschein and S. Yankielowicz, Supergravity and the large $N$ limit of theories with sixteen supercharges, 'Ph [hep-th/9802042in.

[30] G. Horowitz and A. Strominger, Black strings and p-branes,

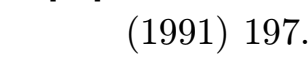

[31] P. Van Nieuwenhuizen, The complete mass spectrum of $d=11$ supergravity compact-

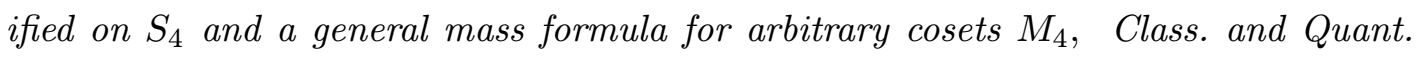
:-

[32] M.J. Teper, Physics from lattice: glueballs in QCD; topology; $S U(N)$ for all $N$, hep-1at/9711011:

[33] C. Morningstar and M. Peardon, Efficient Glueball Simulations on Anisotropic Lat-

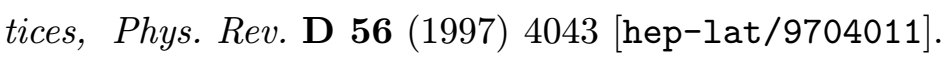

[34] O. Aharony, Y. Oz and Z. Yin, $M$ Theory on $A d S_{p} \times S^{11-p}$ and superconformal field theories, 'Phys. Lett. B-430 (1998) 87, hep-th/9803051'.

[35] H. Ooguri, J. Tannenhauser and H. Robins, Glueballs and their Kalaluza-Klein cousin,

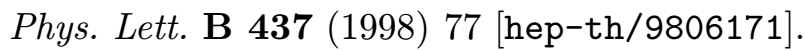

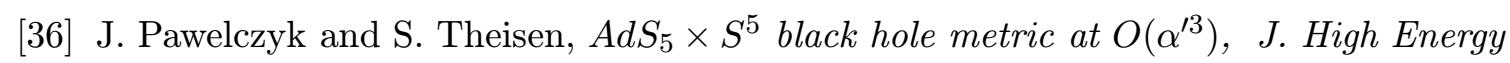

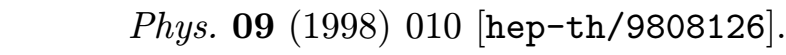

[37] Note added to [1] $1 \overline{7}]$ on August 19, 1998. 